\title{
INFECTIOUS DISEASES, NSW: OCTOBER 1998
}

\section{TRENDS}

As shown by Figure 1, this spring has so far been relatively quiet, at least for most seasonal communicable diseases. The incidence of gonorrhoea remains a concern, however, particularly among gay men (see report p. 120).

\section{CHANGES TO THE NSW NOTIFIABLE DISEASES SCHEDULE}

On 25 September 1998, five new infectious diseases were added to the list of conditions that laboratories are required to report to local public health units. These are:

1. Chancroid, an acute bacterial sexually transmitted disease (STD), which is characterised by single or multiple painful necrotising ulcers. It is most prevalent in tropical and subtropical regions and is rare in Australia. It is caused by Haemophilus ducreyi.

2. Chlamydia infection, one of the most common bacterial STDs in Australia. It causes urethritis in males, and mucopurulent cervicitis in females. Infection in pregnancy can result in conjunctival and pneumonic infection of the newborn. It is caused by Chlamydia trachomatis.

3. Donovanosis, a chronic, and (if untreated) progressively destructive, ulcerative bacterial STD, which is endemic among Aboriginal people and Torres Strait Islanders in some central and tropical regions of Australia. It is caused by Calymmatobacterium granulomatis.

4. Lymphogranuloma venereum (LGV), a bacterial STD characterised by genital lesions and regional lymph node suppuration with inflammation that may extend to adjacent tissues, or proctitis. It is rare in Australia. It is caused by LGV serovars of Chlamydia trachomatis.

5. Giardiasis, a parasitic infection, which causes gastroenteritis, spread by the faecal-oral route (mainly person-to-person or from contaminated water or food). It is caused by Giardia lamblia.

The initiation of public health surveillance for these conditions brings NSW into line with other States, will provide a better understanding of their epidemiology in NSW, and will inform the planning of evaluation of prevention and treatment programs. While giardiasis is transmitted by a variety of vehicles, of prime concern for communities are outbreaks caused by contaminated water supplies. The surveillance of this organism will provide a new tool for better identifying potential water-borne outbreaks.

Except for clusters of cases, or evidence of unusual exposures, public health unit staff will not generally follow up individual case notifications of these diseases. Clinicians seeking advice about the diagnosis, management or contact tracing of any STDs (including those listed above) are encouraged to contact their local Area sexual health clinic or their local public health unit (listed on page 113).

\section{Hospital infection surveillance system}

The NSW Health Department has funded the University of NSW Hospital Infection Epidemiology Unit to run a pilot investigation of the NSW Hospital Infection Surveillance System (HISS). The project coordinator is Dr Mary-Louise McLaws. The HISS will result in the development of standardised, valid and reliable surveillance data.

The establishment of the pilot for the HISS is based on the recommendations of the first national prevalence study of nosocomial and community-acquired infections that was undertaken in 1984 by McLaws and the NSW Nosocomial Infection Taskforce. ${ }^{1}$

The HISS complements a range of infection-control programs already established in NSW, including:

- the NSW health infection control policy (currently being revised)

- the Nosocomial Infection Outcome Indicator Project

- the NSW Infection Control Resource Centre

- the NSW 24- hour needle stick injury hotline

- regulated infection-control standards for medical practitioners, nurses, dentists, podiatrists, physiotherapists and dental technicians

- distribution of the NSW infection control training and information resources kit

- the NSW Infection Control Advisory Group.

The pilot investigation will involve collection of standardised surveillance data at 10 pilot sites for 12 months from November 1998. These sites are Prince of Wales, John Hunter, St George, New Children's, Wollongong, Westmead, Albury Base, Tamworth Base, Mona Vale and Nepean hospitals.

Data will be collected to describe local surgical infections, intravascular device-related bacteraemia infections, respiratory syncytial virus, rotavirus, multiple-resistant organisms, including methicillin-resistant Staphylococcus aureus (MRSA), vancomycin-resistant enterococci (VRE), extended-spectrum beta-lactamase-producing enterics (ESBL), gentamicin-resistant enteric bacteria and others.

Sites have been equipped with hand-held computers, and specialised software has been developed for the project. Analysis of the data will be undertaken by the HISS Coordination Unit and a report will be produced using the aggregate data on rates of infection and on the implications of the pilot for continuing surveillance. Individual sites 


\section{FIGURE1}

REPORTS OF SELECTED INFECTIOUS DISEASES, NSW, JANUARY 1994 TO SEPTEMBER 1998, BY MONTH OF ONSET

These are preliminary data: case counts in recent months may increase because of reporting delays
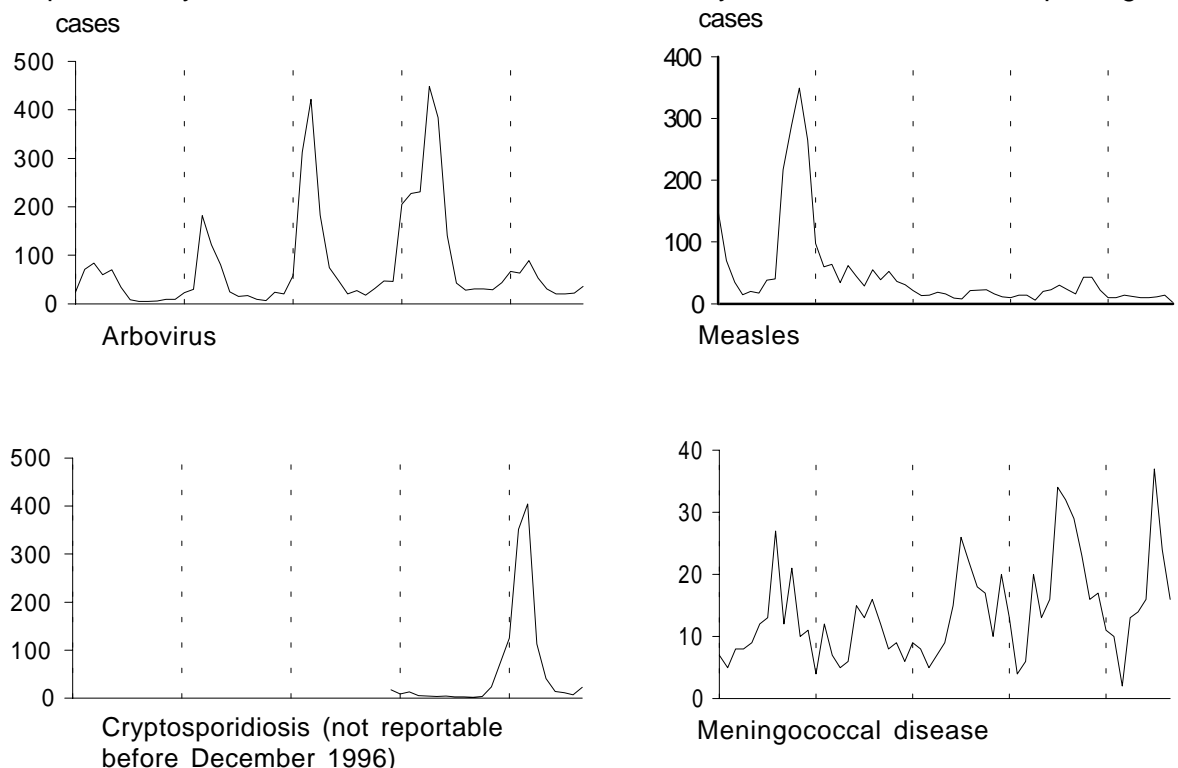

before December 1996)

Meningococcal disease
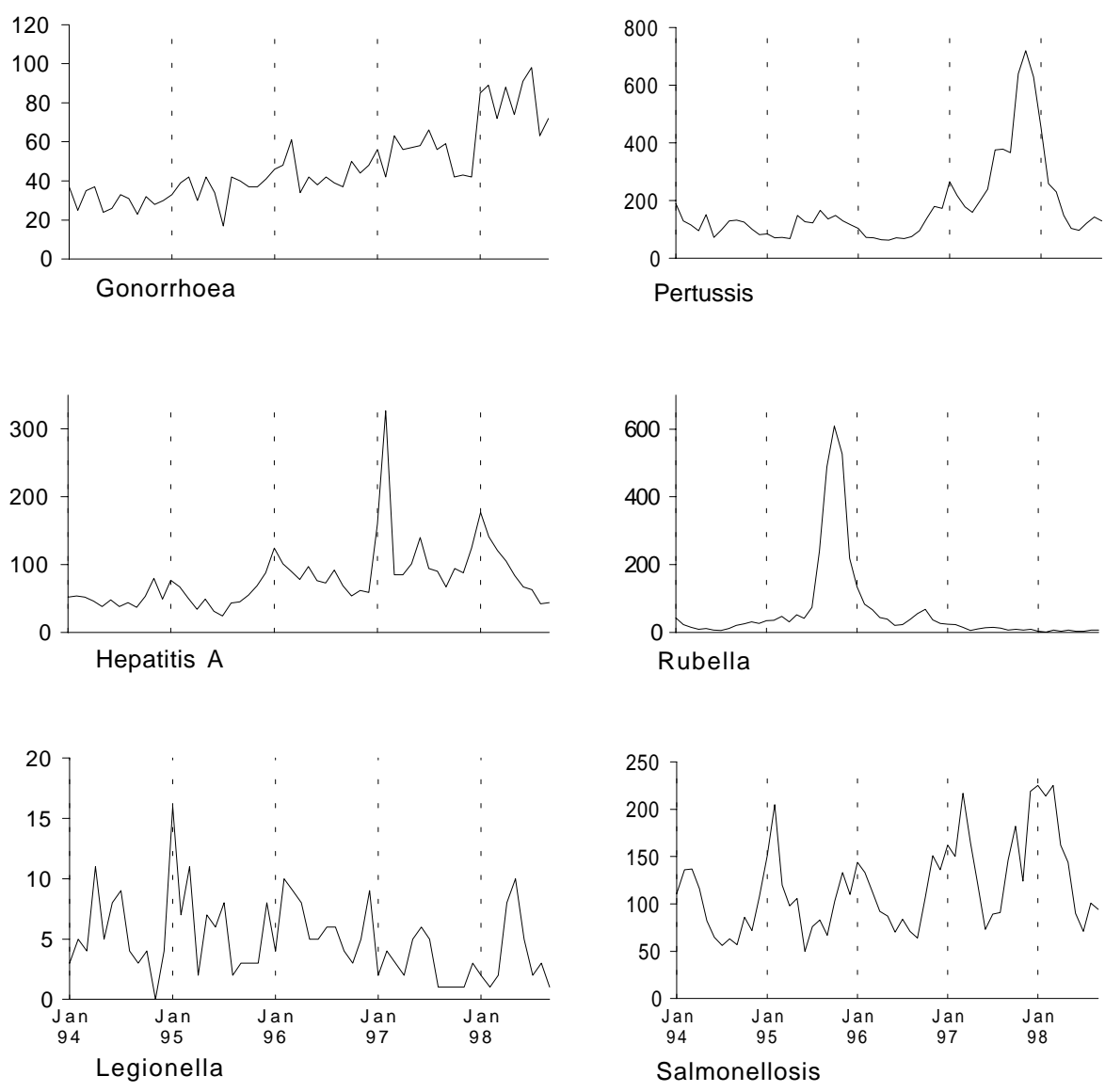
will be provided with the results of the analysis of their own data.

\section{REFERENCE}

1. McLaws ML et al. The prevalence of nosocomial and community acquired infections in Australian hospitals. Med J Aust 1988; 149: 582-590.

\section{INCREASE IN GONORRHOEA}

Gonococcal surveillance NSW-quarterly report:

1 April-30 June 1998

World Health Organization Collaborating Centre for STD and HIV, Microbiology Department, Prince of Wales Hospital, Randwick

\section{Summary}

Two features have been prominent in quarterly reports of Neisseria gonorrhoeae gonococcus isolate surveillance in NSW over the past year and a half: a continuing increase in the number of isolates referred and a high proportion of isolates resistant to the quinolone group of antibiotics. Both these trends were maintained in the April-June quarter of 1998.

In this quarter, 322 isolates of gonococcus were examined, almost 50 per cent more than in the same period last year. Numbers seen in the six months to 30 June were higher than the total number of isolates seen in the whole of either 1994 or 1995 . There has been a 20 per cent increase in the number of gonococcal isolates in NSW each year since 1994.

Resistance to the oral antibiotics available for treating gonococci was also again high. Resistance to the penicillin group was found in more than 40 per cent of all isolates and to the quinolone group in more than 5 per cent of all strains. Quinolone-resistant $N$. gonorrhoeae (QRNG) were until now found only in strains transmitted by heterosexual contact. In this quarter QRNG were also detected among gonococci transmitted by male homosexual contact.

\section{Numbers of gonococcal isolates}

In this quarter, there was again a substantial increase in the number of gonococcal isolates, compared with historical data.

In the three months to 30 June 1998, 322 isolates were available for further examination (217 isolates in 1997 and 136 in 1996).

There were 294 isolates were from infected males, a marked increase over the 200 isolates from males in the same period in 1997. The number of isolates from females increased to 28 from 17 in 1997.

There were 65 isolates from the anorectum or pharynx of male patients in the June quarter of 1998 compared with 18 in 1997 (Table 3). Isolates were obtained from males and females in the ratio of 10.5:1 (11.8:1 in the June quarter in 1997). The 47 male rectal isolates comprised 16 per cent of gonococci isolated from men (6 per cent in 1997) and the 18 pharyngeal isolates 6 per cent (3 per cent in 1997).

\section{Antibiotics available for treatment of gonorrhoea}

The following antibiotics are available for single-dose treatment of gonorrhoea in NSW:

- oral penicillins (including penicillin, ampicillin and amoxycillin, not recommended)

- third-generation cephalosporins, which include injectable ceftriaxone and injectable cefpodoxime (soon to be discontinued)

- quinolones, which include ciprofloxacin, norfloxacin, enoxacin and ofloxacin

- injectable spectinomycin

\section{Antibiotic sensitivity patterns \\ Penicillins}

Of the strains tested in the June quarter of 1998, 147 (45.6 per cent) were penicillin resistant, 16 (5 per cent) being penicillinase-producing $N$. gonorrhoeae (PPNG) and 131 (40.6 per cent) resistant by chromosomal mechanisms (CMRNG). The former were isolated from patients who contracted their infection locally (3) and overseas (7), and

\section{TABLE 3}

\section{SITES OF INFECTION WITH NEISSERIA} GONORRHOEAE, SYDNEY, 1 APRIL TO 30 JUNE 1998 (FROM LABORATORY DATA)

\begin{tabular}{lr|}
\hline Site & No. \\
\hline Male & 228 \\
Urethra & 18 \\
Pharynx & 47 \\
Anorectum & 1 \\
Disseminated & 0 \\
Eye & 1 \\
Other & 294 \\
Total & \\
Female & 26 \\
Endocervix or vagina & 2 \\
Pharynx & 0 \\
Anorectum & 0 \\
Disseminated & 0 \\
Eye & 0 \\
Other & 0 \\
Total &
\end{tabular}

Source: WHO Collaborating Centre for STD and HIV 
the location was not specified for another 6. Approximately 40 per cent of isolates were penicillin resistant by one or more mechanisms.

Because nearly half of all the gonococci examined were resistant to the penicillins, the use of penicillin-based treatment regimens (including amoxycillin and ampicillin) would result in a significant proportion of treatment failures.

\section{Ceftriaxone}

All isolates examined were sensitive to this injectable third-generation antibiotic, which has retained its activity against gonococci over many years. A small number of isolates showed some increase in minimum inhibitory concentration (MIC). Although not clinically relevant at this stage, this phenomenon is indicative of trends in susceptibility that may cause future problems. Continued monitoring is warranted. Isolates would also be susceptible to other injectable third-generation agents but not to earlygeneration oral cephalosporins.

\section{Spectinomycin}

All strains were susceptible in vitro to this injectable antibiotic.

\section{Quinolone group}

A significant proportion of isolates were resistant to quinolone antibiotics (ciprofloxacin, norfloxacin, ofloxacin, enoxacin), although the proportion of QRNG was not as high as in the March quarter of 1998

The $18 \mathrm{QRNG}$ isolated represented 5.6 per cent of all isolates. Eleven of the 18 strains isolated this quarter manifested high levels of resistance to this antibiotic group (MIC of ciprofloxacin $1 \mathrm{mg} / \mathrm{L}$ or less, 3.4 per cent of all isolates). The MICs of the QRNG ranged from 0.125 to $16 \mathrm{mg} / \mathrm{L}$. Thirtytwo (14.7 per cent) isolates were quinolone-resistant in the June quarter of 1997. Details about geographic acquisition of QRNG were available for only 6 of the 18 patients with QRNG in this quarter. Of these, three cases were acquired locally and three overseas.

Strain subtyping on the QRNG also indicates a further change in the distribution of QRNG subtypes. A recent phenomenon has been the identification of a number of apparent clusters of QRNG in local isolates from heterosexually acquired gonorrhoea. These particular subtypes are no longer as prominent as in previous quarters. QRNG have now also been identified in gonorrhoea acquired by homosexual contact.

\section{Tetracyclines}

The tetracycline group of antibiotics is not recommended for the treatment of gonococcal infection. All of the agents mentioned above can be administered as a single dose to ensure patient compliance, whereas the tetracycline-based treatment regimens are multiple-dose therapies, use of which also contributes to antibiotic resistance. In NSW in each quarter, several treatment failures are recorded for patients who receive only tetracycline therapy when presenting with a urethral discharge. A pattern of suppression of symptoms followed by recrudescence some time after completion of therapy has been seen repeatedly.

The most recent examination of tetracycline-resistance patterns indicated that about 30 per cent of NSW isolates were resistant. In addition, a form of high-level plasmid resistance to the tetracyclines has emerged in the past decade. Isolates possessing this plasmid are identified as tetracycline-resistant $N$. gonorrhoeae (TRNG). In this quarter, 21 cases of TRNG were detected (6.5 per cent of all isolates).

\section{Editorial comment}

NSW gonorrhoea notifications have shown an increasing trend over the past few years (Figure 1). The number of notifications for 1998 to the end of August (569) was 42 per cent higher than for the same period in 1997 (401), and 162 per cent higher than for the same period in 1994 (217). Increases have been more marked in males over 25 years of age from the South Eastern Sydney Area. Since 1994 the proportion of cases in males have increased from 83 per cent to 92 per cent. Increases were seen for almost all age groups; however, there was a tendency towards larger increases in older age groups: 38 per cent of cases were aged under 25 years in 1994, compared with 23 per cent in 1998. The proportion of notifications from South Eastern Sydney increased from 40 per cent in 1994 to 50 per cent in 1998.

In response to this increase, an expert committee was recently convened by the NSW Health Department to recommend a plan of action. Fact sheets for medical practitioners and patients are being distributed by public laboratories with all new gonorrhoea diagnoses. The fact sheets provide guidelines for treatment and contact tracing and provide information about the services provided by sexual health centres. Also, the AIDS Council of NSW has planned campaigns, to commence shortly, focusing on safe sex and hepatitis A immunisation among homosexual men. 


\section{TABLE 4}

INFECTIOUS DISEASE NOTIFICATIONS RECEIVED IN SEPTEMBER 1998 BY AREA HEALTH SERVICES

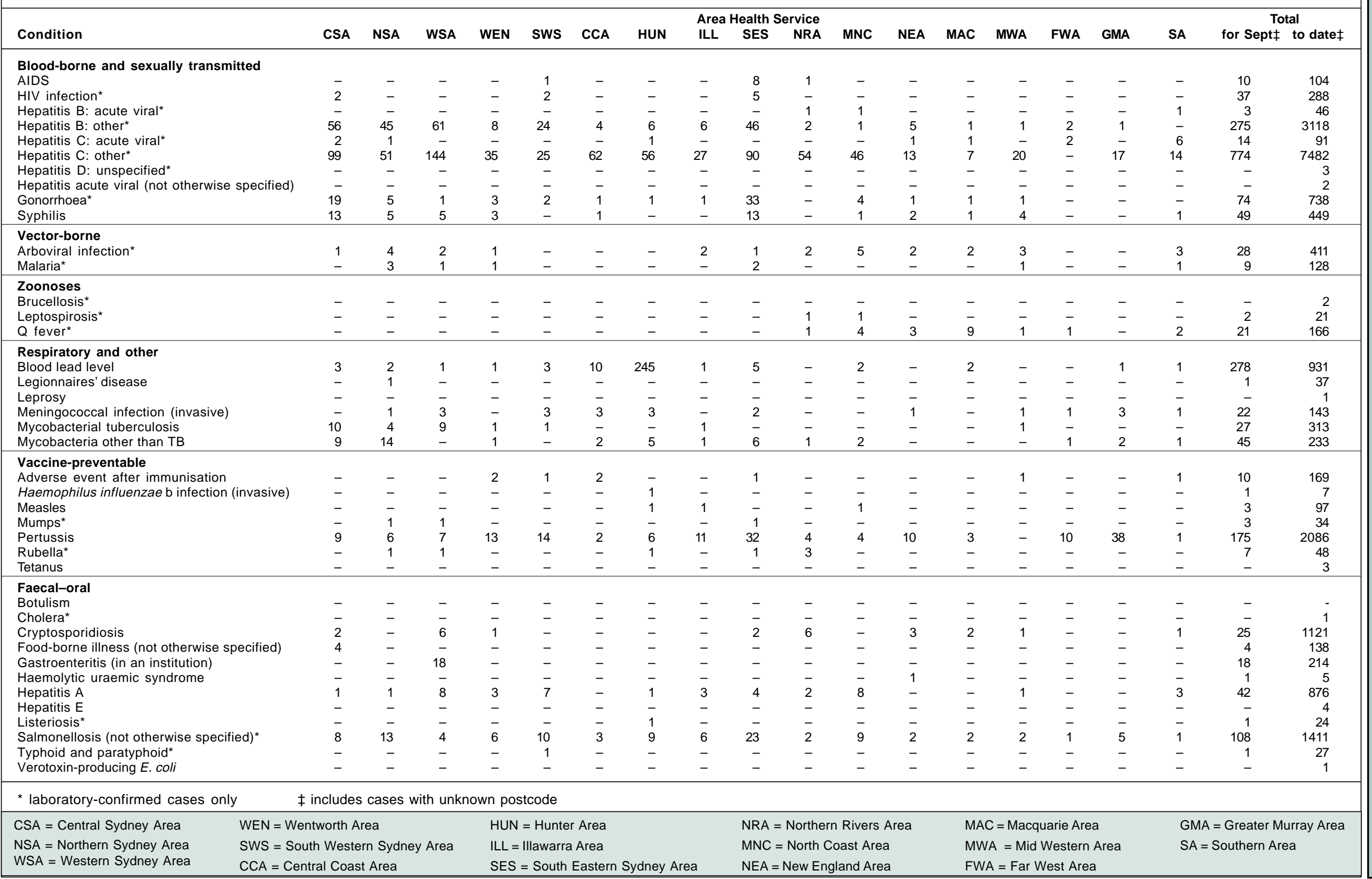

